\title{
IMPACTO DE LA CLIMATOLOGÍA SOBRE EL NIVEL SÉRICO dE ANTíGeNO ESPECIFICO PROSTÁTICO (PSA).
}

\author{
Marcos Luján Galán, Carlos Pascual Mateo, Nuria Rodríguez García, José María García \\ Mediero, Tomás Pascual Durán', Álvaro Paez Borda² y Antonio Berenguer Sánchez.
}

Servicio de Urología. Hospital Universitario de Getafe. Getafe. Madrid.

Servicio de Bioquímica'. Hospital Universitario de Getafe. Getafe. Madrid.

Servicio de Urología². Hospital de Fuenlabrada. Madrid. España.

\begin{abstract}
Resumen.- OBJETIVO: Analizar el impacto de la estación climática y de ciertos parámetros meteorológicos sobre el nivel de PSA sérico en varones sin cáncer de próstata.

MÉTODOS: Estudio retrospectivo que incluye registros de la rama Española del European Randomized Study of Screening for Prostate Cancer (ERSPC). Los criterios de exclusión fueron el diagnóstico de cáncer de próstata, presentar PSA $>=10 \mathrm{ng} / \mathrm{ml}$, o bien PSA $>=3$ $\mathrm{ng} / \mathrm{ml}$ y/o anomalías en el tacto rectal a menos que se disponga de una biopsia prostática negativa para malignidad. Se investigó la relación univariante entre nivel de PSA y estación climática, así como varios parámetros meteorológicos. Se empleó un modelo multivariante de regresión logística para identificar predictores independientes de la obtención de un nivel de PSA >=3 $\mathrm{ng} / \mathrm{ml}$.
\end{abstract}

RESULTADOS: Un total de 2.147 varones fueron incluidos en el estudio. Las medianas de edad y PSA fueron 57 años y $0,9 \mathrm{ng} / \mathrm{ml}$ respectivamente. Se observó una tendencia no significativa hacia niveles de PSA más elevados durante las estaciones de otoño e invierno. El modelo multivariante de regresión logística identificó únicamente las temperaturas máxima $(p<0,001)$, mínima $(p=0,001)$ y la edad $(p<0,001)$ como predictores independientes de un nivel de PSA $>=3 \mathrm{ng} / \mathrm{ml}$. Los niveles medios de PSA ajustado a la edad en los rangos de temperatura máxima $<=15^{\circ} \mathrm{C}, 16-20^{\circ} \mathrm{C}, 21-25^{\circ} \mathrm{C}$ y $>=26^{\circ} \mathrm{C}$ fueron $1,25,1,20,1,17$ y $1,09 \mathrm{ng} / \mathrm{ml}$ respectivamente.

CONCLUSIONES: Los niveles de PSA sérico son ligeramente más elevados durante condiciones de frío climático. Debido a la pequeña magnitud de esta elevación, no recomendamos cambiar la indicación de biopsia prostática basándonos únicamente en factores climáticos.

Palabras clave: Antígeno específico prostático. Cáncer de próstata. Climatología.

Summary.- OBJECTIVES: To analyze the impact of season and weather parameters on serum PSA values in men without prostate cancer.

METHODS: Retrospective study including medical records from the Spanish arm of the European Randomized Study of Screening for Prostate Cancer (ERSPC). Exclusion criteria: prostate cancer diagnosis, PSA $>=10 \mathrm{ng} / \mathrm{ml}$, or PSA $>=3 \mathrm{ng} / \mathrm{m} /$ and $/$ or digital rectal examination abnormalities unless a negative prostate biopsy was provided. 
Univariate relationships between PSA value, season and several weather parameters were assessed. A multivariate logistic regression model was used to identify independent predictors of a PSA value $>=3 \mathrm{ng} / \mathrm{m}$.

RESULTS: A total of 2,147 men entered into the study. Median age and PSA level were 57 years and 0.9 $\mathrm{ng} / \mathrm{ml}$ respectively. A non-significant trend to higher PSA levels was observed during autumn and winter. Multivariate logistic regression analysis identified only maximum temperature $(p<0.001)$, minimum temperature $(p=0.001)$ and age $(p<0.001)$ as independent predictors of a PSA value $>=3 \mathrm{ng} / \mathrm{ml}$. Mean age-adjusted PSA levels at maximum temperatures of $<=15^{\circ} \mathrm{C}, 16-20^{\circ} \mathrm{C}$, $27-25^{\circ} \mathrm{C}$ and $>=26^{\circ} \mathrm{C}$ were $1.25,1.20,1.17$ and $1.09 \mathrm{ng} / \mathrm{m} /$ respectively.

CONCLUSIONS: PSA levels are slightly higher during cold weather conditions. Because of the small magnitude of this PSA increment we do not recommend to change biopsy indication based solely on climatic parameters.

Keywords: Prostate-specific antigen. Prostate cancer. Weather climate.

\section{INTRODUCCIÓN}

El antígeno específico prostático (PSA) se ha convertido en el marcador más utilizado para la detección del cáncer de próstata (CaP) (1). Un nivel elevado de PSA implica procedimientos invasivos como la biopsia prostática. Por desgracia, el rendimiento diagnóstico de este marcador es limitado debido a que también pueden presentarse niveles elevados de PSA en presencia de entidades exentas de malignidad, como la hiperplasia benigna de próstata (HBP), principalmente en rangos de PSA intermedios (3 - 10 $\mathrm{ng} / \mathrm{ml}$ ). Por este motivo se ha intentado cuantificar la variabilidad asumible de los niveles de PSA en pacientes sin cáncer (2). También se ha estudiado la repercusión de diversos factores, como la existencia de prostatitis, HBP, eyaculación reciente, realización de tacto rectal, cateterización o instrumentación uretral, biopsia prostática y otros (3 - 5) sobre los niveles de PSA en pacientes en los que se ha excluido la presencia de CaP.

Por otro lado, es bien conocido en el ámbito urológico que los pacientes que sufren de HBP y sintomatología prostática son más proclives a una exacerbación de sus síntomas durante la exposición a climas y estaciones fríos. No obstante, han sido pocos los estudios que han revisado la variación estacional de los niveles de PSA (6) y no conocemos que haya sido nunca estudiada la relación entre los niveles de PSA séricos y los parámetros meteorológicos.
En el presente estudio analizamos el impacto estacional y de varios parámetros meteorológicos sobre el valor de PSA sérico en varones sin cancer de próstata.

\section{MATERIAL Y MÉTODOS}

Este estudio retrospectivo se ha llevado a cabo con varones reclutados en la rama Española del European Randomized Study of Screening for Prostate Cancer (ERSPC), estudio que pretende demostrar una diferencia en la mortalidad por CaP entre los dos brazos del estudio: brazo screening (varones sometidos a tests diagnósticos y tratamiento si procede) y brazo control (sin realización de tests), randomizados 1:1. El presente trabajo se ha llevado a cabo partiendo de 2.296 varones con edades comprendidas entre los 45 y 71 años reclutados en el brazo screening desde 1/1/1997 hasta 31/12/1999 (primera ronda del estudio). Se realizó determinación del nivel de PSA sérico. Con el objeto de reducir al máximo el riesgo de que estos pacientes albergaran $\mathrm{CaP}$, se excluyeron (además de todos los casos en los que se diagnosticó (aP) todos los casos con PSA > = $10 \mathrm{ng} /$ $\mathrm{ml}$. Además, los individuos con PSA > = $3 \mathrm{ng} / \mathrm{ml}$ y/o anomalías en el tacto rectal fueron también excluidos a menos que hubieran presentado una biopsia prostática negativa para malignidad.

El presente estudio se llevó a cabo en el Area Sanitaria 10 de la Comunidad de Madrid, que comprende una población de unos 300.000 y las localidades de Getafe, Parla y Pinto (entre otras) al sur de la ciudad de Madrid, a 646 metros sobre el nivel del mar y aproximadamente a unos $400 \mathrm{~km}$ de la costa más cercana. Madrid presenta un clima continental con inviernos fríos y veranos calurosos y secos (las temperaturas máximas pueden alcanzar hasta $44^{\circ} \mathrm{C}$ durante los meses de Julio y Agosto).

Se analizó la relación univariante entre en nivel de PSA sérico y la estación del año mediante el test no paramétrico de Kruskal-Wallis test. La correlación entre los niveles de PSA y los diversos parámetros climáticos se estudió mediante el coeficiente de correlación de Spearman. Las distintas variables climáticas estudiadas fueron las temperaturas diarias máxima y mínima (en grados Celsius), humedad relativa del aire (porcentaje), cantidad de precipitación $(\mathrm{mm})$ y número de horas de sol. Estos datos se obtuvieron del Instituto Nacional de Metereología en la forma de tablas con las mediciones diarias (tomadas en la Base Aérea de Getafe, Madrid) de los parámetros mencionados ajustados estrictamente con el día y el lugar donde las determinaciones de PSA fueron realizados (Hospital de Getafe, Madrid). Debido a la 
conocida relación entre los niveles de PSA y la edad, para el análisis univariante se ajustaron los valores de PSA de acuerdo con la siguiente fórmula:

PSA ajustado por edad $=$ PSA $\times$ EMP / edad

EMP = edad media de la población final estudiada $(n=2.147): 57,5$ años.

Considerando las mencionadas variables, empleamos un modelo multivariante de regresión logística para identificar qué factores eran capaces de predecir de modo independiente la obtención de un nivel de PSA anormal $>=3 \mathrm{ng} / \mathrm{ml}$, nivel de corte empleado en nuestro Centro para recomendar una biopsia prostática).

El análisis estadístico se realizó con la ayuda del paquete estadístico SPSS 11.0 (SPSS Inc. Chicago, IL, USA). El nivel de significación estadística se alcanzó cuando $p<0.05$.

\section{RESULTADOS}

De los 2.296 varones reclutados, un total de 2.147 fueron elegibles para el presente estudio. La edad osciló entre 46 y 71 años, mediana 57 , media 57,5 , error estándar de la media (EEM) 0,118. Los valores de PSA de oscilaron entre 0,1 y $9,6 \mathrm{ng} / \mathrm{ml}$, mediana 0,9 , media 1, 19, EEM 0,024.

En la Tabla I resumimos los valores de los parámetros climáticos encontrados en nuestro área durante el periodo del estudio.

Se analizó la relación entre la estación climática y los valores de PSA, encontrando una tendencia no significativa $(p=0,092)$ hacia niveles de PSA ajustados a la edad más altos durante las estaciones más frías (otoño e invierno) (Figura 1).

El análisis univariante (Tabla II, Figura 2) mostró una correlación negativa significativa entre

TABLA I. VALORES MEDIOS DE LOS PARÁMETROS METEOROLÓGICOS OBASERVADOS DURANTE EL PERIODO DE ESTUDIO. LOCALIZACIÓN: BASE AEREA DE GETAFE. MADRID. ESPAÑA.

\begin{tabular}{|lcccccc|}
\hline & $\mathbf{N}$ & $\begin{array}{c}\text { Temperatura } \\
\text { máxima }\left({ }^{\circ} \mathbf{C}\right)\end{array}$ & $\begin{array}{c}\text { Temperatura } \\
\text { mínima }\left({ }^{\circ} \mathbf{C}\right)\end{array}$ & $\begin{array}{c}\text { Humedad } \\
\text { relativa (\%) }\end{array}$ & $\begin{array}{c}\text { Precipitación } \\
(\mathbf{m m})\end{array}$ & Horas de sol \\
\hline Primavera & 646 & 22,8 & 10,6 & 57,0 & 1,5 & 8,6 \\
\hline Verano & 340 & 32,3 & 17,6 & 35,6 & 0,0 & 12,5 \\
\hline Otoño & 467 & 15,4 & 5,8 & 73,7 & 2,5 & 4,1 \\
\hline Invierno & 694 & 15,9 & 3,8 & 68,2 & 0,5 & 7,3 \\
\hline Total & 2147 & 20,5 & 8,5 & 60,9 & 1,1 & 7,8 \\
\hline
\end{tabular}

\section{TABLA II. ANÁLISIS UNIVARIANTE. CORRELACIÓN ENTRE LOS VALORES DE PSA, EDAD Y PARÁMETROS CLIMÁTICOS}

\begin{tabular}{|l|c|c|}
\hline & Coeficiente de correlación de Spearman & P \\
Edad & 0,219 & $<0,001$ \\
\hline Temperatura máxima & $-0,050$ & 0,022 \\
\hline Temperatura mínima & $-0,033$ & 0,127 \\
\hline Humedad relativa & 0,085 & $<0,001$ \\
\hline Precipitación & 0,045 & 0,036 \\
\hline Horas de sol & $-0,048$ & 0,027 \\
\hline
\end{tabular}




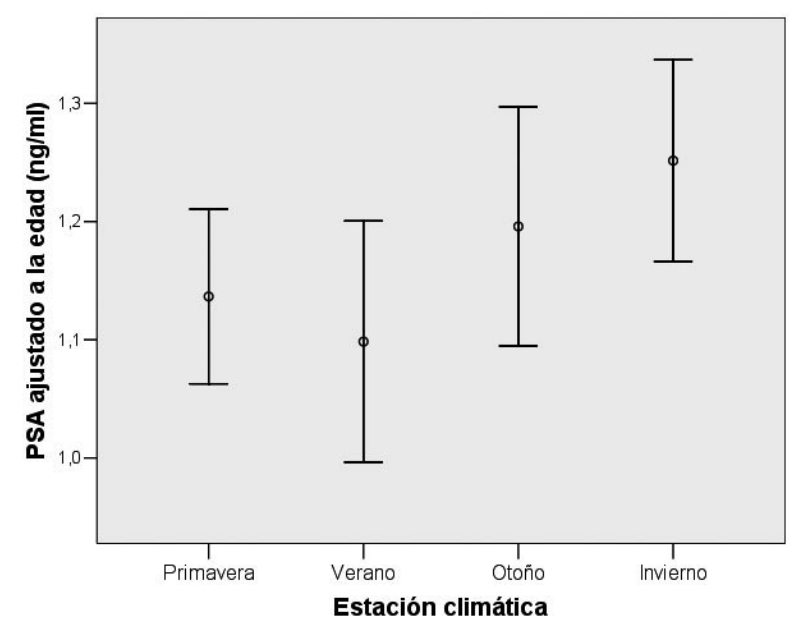

FIGURA 1. Gráfico de barras de error mostrando valores medios e intervalos de confianza (95\%) para valores de PSA ajustado a la edad con respecto a cada estación climática. Test de Kruskal-Wallis $p=$ 0,092 (no significativo).

los niveles de PSA y las variables temperatura máxima y horas de sol. Se encontró correlación positiva significativa entre los niveles de PSA y la edad, humedad relativa del aire y cantidad de precipitación.

Tras el análisis multivariante (regresión logística), únicamente las temperaturas máxima $(p<0.001)$ y mínima $(p=0.001)$, así como la edad $(p<0.001)$ fueron identificados como predictores independientes de la obtención de un valor de PSA $>=3 \mathrm{ng} / \mathrm{ml} / \mathrm{y}$, por tanto, pasando a ser candidatos para biopsia prostática). En la Tabla III se muestra la magnitud del cambio de PSA de acuerdo a los distintos rangos de temperatura máxima y mínima.

\section{DISCUSIÓN}

Es sobradamente conocido que los pacientes con HBP y síntomas prostáticos son advertidos por sus médicos acerca de un posible empeoramiento de su sintomatología ante la exposición a estaciones o climas fríos. Desafortunadamente existen pocos datos acerca de la repercusión estacional en los niveles de PSA [6], y no existe evidencia a favor ni en contra acerca de la correlación entre dichos niveles y los distintos parámetros climáticos. En nuestro estudio, el análisis multivariante mostró una modesta pero significativa relación entre la temperatura climática y los niveles de PSA demostrando así que las bajas temperaturas pueden contribuir a un incremento de los niveles de PSA séricos incluso por encima de los niveles de corte para biopsia prostática. No obstante, dicho incremento es de una magnitud pequeña (Tabla III). Por ello pensamos que incrementos importantes de PSA nunca deben ser atribuidos a la variabilidad inducida por factores meteorológicos.

Sin duda habría sido interesante analizar los niveles de PSA y valores de temperatura en conjunto con información acerca de los síntomas prostáticos (por ejemplo mediante cuestionario IPSS). Por desgracia, esto no fue posible debido al carácter retrospectivo del estudio.
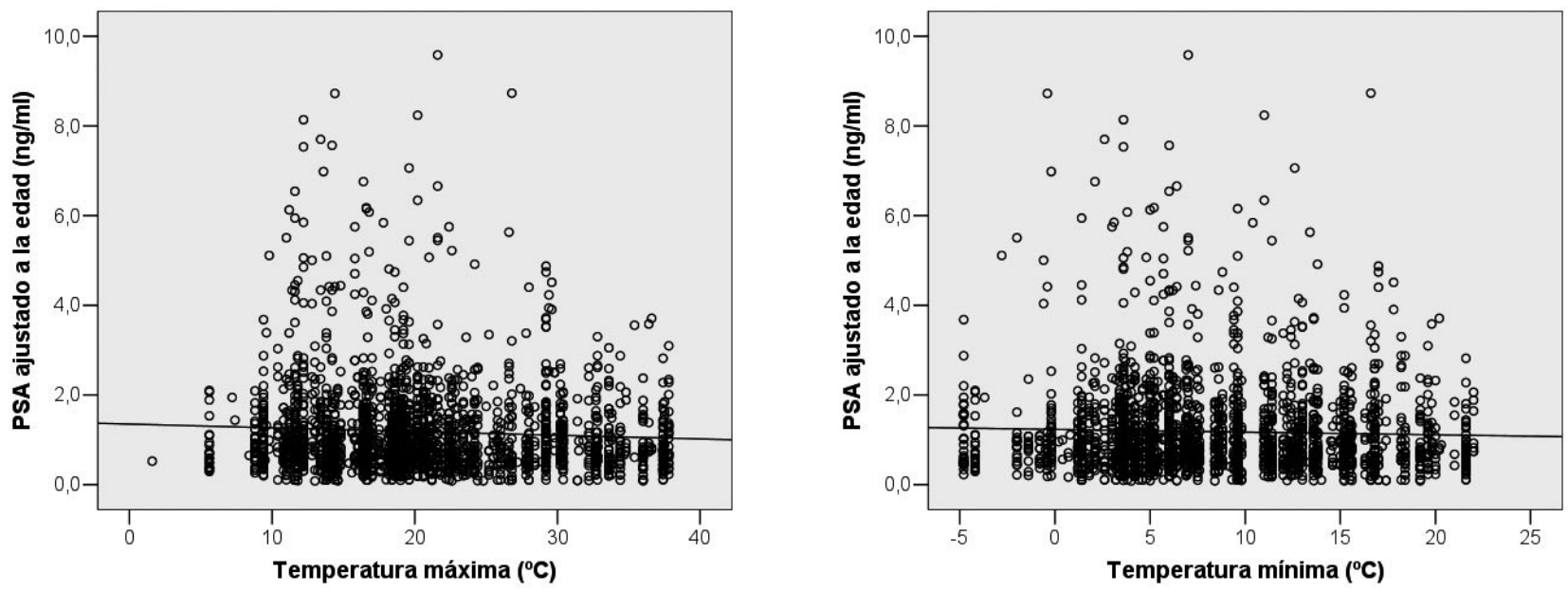

FIGURA 2. Gráficos que muestran la correlación univariante entre el nivel de PSA ajustado a la edad y las temperaturas climáticas máxima (Figura 2a: $\rho=-0,050, p=0,022$ ), y mínima (Figura 2b: $\rho=-0,033, p=0,127$ ). 
TABLA IIIa. NIVELES DE PSA AJUSTADO A LA EDAD SEGÚN RANGOS DE TEMPERATURA MÁXIMA.

\begin{tabular}{|c|c|c|c|}
\hline \multirow{2}{*}{$\begin{array}{l}\text { Temperatura máxima } \\
\qquad\left({ }^{\circ} \mathrm{C}\right)\end{array}$} & \multirow[b]{2}{*}{$\mathbf{N}$} & \multicolumn{2}{|c|}{ Nivel de PSA ajustado a la edad } \\
\hline & & $\begin{array}{c}\text { Media (EEM) } \\
\text { (ng/ml) }\end{array}$ & $\begin{array}{c}\text { Varones con PSA > }=3 \\
\mathrm{ng} / \mathrm{ml}\end{array}$ \\
\hline$<=15$ & 592 & $1,25(0,05)$ & $39(6,6 \%)$ \\
\hline $16-20$ & 597 & $1,20(0,04)$ & $33(5,5 \%)$ \\
\hline $21-25$ & 417 & $1,17(0,05)$ & $14(3,4 \%)$ \\
\hline$>=26$ & 541 & $1,09(0,04)$ & $22(4,1 \%)$ \\
\hline Total & 2147 & $1,18(0,02)$ & $108(5,0 \%)$ \\
\hline
\end{tabular}

EEM = error estándar de la media.

TABLA IIIa. NIVELES DE PSA AJUSTADO A LA EDAD SEGÚN RANGOS DE TEMPERATURA MÍNIMA.

\begin{tabular}{|c|c|c|c|}
\hline \multirow{2}{*}{$\begin{array}{l}\text { Temperatura máxima } \\
\qquad\left({ }^{\circ} \mathrm{C}\right)\end{array}$} & \multirow[b]{2}{*}{$\mathbf{N}$} & \multicolumn{2}{|c|}{ Nivel de PSA ajustado a la edad } \\
\hline & & $\begin{array}{c}\text { Media (EEM) } \\
\text { (ng/ml) }\end{array}$ & $\begin{array}{c}\text { Varones con PSA >=3 } \\
\mathrm{ng} / \mathrm{ml}\end{array}$ \\
\hline$<=5$ & 734 & $1,18(0,04)$ & $31(4,2 \%)$ \\
\hline $6-10$ & 645 & $1,24(0,04)$ & $40(6,2 \%)$ \\
\hline $11-15$ & 372 & $1,19(0,05)$ & $21(5,6 \%)$ \\
\hline$>=16$ & 396 & $1,08(0,05)$ & $16(4,0 \%)$ \\
\hline Total & 2147 & $1,18(0,02)$ & $108(5,0 \%)$ \\
\hline
\end{tabular}

EEM = error estándar de la media.

Somos conscientes de que la temperatura del aire exterior puede no reflejar completamente las condiciones de temperatura reales que cada paciente ha sufrido, y distintos factores socioeconómicos pueden tener influencia sobre la exposición climática. No obstante, nuestro trabajo ha demostrado que la temperatura climática tiene realmente un impacto sobre los niveles de PSA y es probable que si se ajustaran los datos obtenidos según nivel socioeconómico, es probable que las diferencias encontradas fueran incluso superiores.

Otro posible factor de confusión podría ser la actividad sexual, aunque este dato no ha sido registrado en nuestro estudio. Se ha comunicado que la eyaculación puede aumentar el nivel de PSA sérico [3]. En cambio, hemos registrado los valores de PSA más altos durante las estaciones más frías (otoño e invierno) y menos proclives a la actividad sexual.

\section{CONCLUSIONES}

Los niveles de PSA son ligeramente superiores cuando su determinación se realiza durante temporadas de frío climatológico. No obstante, debido a la pequeña magnitud de dicho incremento, no recomendamos cambiar las indicaciones de biopsia prostática basándonos únicamente en factores climáticos.

\section{AGRADECIMIENTOS Y SOPORTE FINANCIERO}

Financiado en parte por becas del Fondo de Investigación Sanitaria (FIS) (93/0903, 96/0248, 96/1800, 99/0245 y 02/0732).

Nuestro Centro es miembro participante en el "European Randomized Study of Screening for Prostate Cancer (ERPSC)". 


\section{BIBLIOGRAFÍA y LECTURAS \\ RECOMENDADAS ( ${ }^{*}$ lectura de interés $y^{* *}$ lectura fundamental)}

*1. CHAN, D.W.; BRUZEK, D.J.; OESTERLING, J.E. y cols.: "Prostate-specific antigen as a marker for prostate cancer: a monoclonal and polyclonal immunoassay compared". Clin. Chem., 33: 1916, 1987.

**2. LUJAN, M.; PAEZ, A.; SÁNCHEZ, E. y cols.: "Prostate specific antigen variation in patients without clinically evident prostate cancer". J. Urol., 162: 1311, 1999.

3. HERSCHMAN, J.D.; SMITH, D.S.; CATALONA, W.J.: "Effect of ejaculation on serum total and free prostate-specific antigen concentrations". Urology, 50: 239, 1997.

4. LECHEVALLIER, E.; EGHAZARIAN, C.; ORTEGA, J.C. y cols.: "Effect of digital rectal examination on serum complexed and free prostate-specific antigen and percentage of free prostate-specific antigen". Urology, 54: 857, 1999.

5. LECHEVALLIER, E.; EGHAZARIAN, C.; ORTEGA, J.C. y cols.: "Kinetics of postbiopsy levels of serum free prostate-specific antigen and percent free prostate-specific antigen". Urology, 53: 731, 1999 .

**6. SIMSEK, U.; KUTLU, S.; YAVASÇAOUGLU, I. y cols.: "Seasonal variation of prostatic acid phosphate and prostate-specific antigen in patients without prostatic malignancy". Eur. Urol., 21: 111,1992 